\title{
Influence du dessèchement du sol sur le comportement hydrique et la croissance du chêne pédonculé (Quercus pedunculata Ehrl.) et du frêne (Fraxinus excelsior L.) cultivés en cases de végétation
}

\author{
G. AUSSENAC et G. LEVY * \\ avec la collaboration technique de Y. Llitevre * et F. Willm \\ I.N.R.A., Station de Sylviculture et Production \\ *I.N.R.A. Station de Recherches sur les Sols forestiers \\ Centre de Recherches forestières de Nancy-Champenoux, F 54280 Seichamps
}

\section{Résumé}

Il a été procédé à la comparaison expérimentale du comportement hydrique du chêne pédonculé et du frêne soumis à un stress (pas d'apport d'eau jusqu'à ce que le potentiel de base $\left(\mathbf{P}_{\mathrm{I}}\right)$ atteigne au maximum - 19 bars), dans de grandes cuves de végétation enterrées et sur des plants âgés de 5 et 4 ans respectivement.

Le chêne diminue fortement sa transpiration dès que le sol se dessèche légèrement. alors que le frêne ne l'abaisse sensiblement que pour des réserves hydriques beaucoup plus faibles. En effet, pour une même chute du potentiel hydrique au cours de la journée, la résistance stomatique augmente bcaucoup plus vite chez le chêne que chez lc frêne ; de plus, une augmentation considérable de la résistance stomatique se produit lorsque ce potenticl atteint - 14 bars chez le chêne, mais seulement -30 bars chez le frêne.

Les $P_{\mathrm{B}}$ diminuent ainsi beaucoup plus rapidement, et le sol se dessèche beaucoup plus vite pour le frêne que pour le chêne.

La croissance en hauteur est arrêtée, pour les deux espèces, dès que le $P_{1}$ : dépasse - 11 bars, valeur atteinte bien plus tôt chez le frêne.

Le chêne apparaît donc comme une espèce mieux adaptée que le frêne à des situations non particulièrement favorisées sur le plan hydrique, ce qui lui permet d'avoir une amplitude écologique bien supérieure.

\section{Introduction}

La mise en valeur des taillis et des taillis-sous-futaie de la moitié nord de la France constitue un problème important pour les gestionnaires forestiers. En effet, ces peuplements adaptés aux besoins en bois de construction et en bois de feu de l'économie rurale du siècle dernier ne correspondent plus à la demande actuelle. Parmi les solutions proposées, il est envisagé de favoriser d'autres espèces parallèlement au chêne, notamment les feuillus précieux, et en particulier le frêne. Il est donc nécessaire de 
connaître le comportement de ces essences dans différents types de milieu. Une étude de relations station-production (Devauchelle \& LeVY, 1977) a mis en évidence le rôle fondamental et même unique des conditions d'approvisionnement en eau pour la croissance du frêne dans le Nord-Est de la France. Des résultats tout à fait comparables s'appliquent pour la région Nord-Picardie (LE GOFF \& LEVY, à paraître).

Afin de pouvoir expliquer les résultats de ces études et d'en généraliser les enseignements, il était nécessaire de préciser le comportement hydrique du frêne comparativement à celui du chêne, qui forme la base des peuplements actuels concernés. En effet, ce comportement est encore mal connu; peu de travaux expérimentaux ont en effet été réalisés dans ce domaine, bien que l'on sache de façon empirique que le frêne est probablement plus exigeant en eau que le chêne.

Les peuplements de chêne sont souvent constitués de chêne pédonculé et de chêne sessile. Nous avons choisi de nous intéresser en premier lieu au chêne pédonculé (Quercus pedunculata Ehrl.), car cette espèce paraît au plan écologique plus proche du frêne que ne l'est le chêne sessile.

L'étude (1) a été menée en case de végétation, afin d'utiliser des plants d'une part croissant en conditions climatiques naturelles (mis à part le contrôle du facteur eau) et d'autre part disposant d'un volume de sol suffisamment important pour leur permettre de pousser normalement plusieurs années.

\section{Dispositif expérimental et méthodes d'étude}

L'expérimentation a consisté à comparer le comportement du chêne pédonculé et du frêne confrontés à un dessèchement du sol; des plants maintenus à la capacité au champ servent de témoins.

Le dispositif expérimental a été mis en place à proximité du Centre National de Recherches Forestières, près de Nancy, en mars 1979. Il est constitué de 26 grandes cuves enterrées ayant les dimensions suivantes : diamètre $144 \mathrm{~cm}$, hauteur $100 \mathrm{~cm}$. Les cuves construites en polyéthylène noir sont raccordées à un local enterré, destiné au recueil des eaux de percolation. Elles ont été remplies de la façon suivante : au fond $10 \mathrm{~cm}$ de gravier pour faciliter le drainage, au-dessus $80 \mathrm{~cm}$ de terre sablolimoneuse provenant de l'horizon $A_{1}$ d'un sol brun acide prélevé en forêt. Ce sol a bénéficié d'une fertilisation minérale, par cuve :

- en avril 1980: 41 grammes de patentkali (M, Mg), 18 grammes de superphosphate triple, 82 grammes de phosphate naturel;

- en juin 1980 : 45 grammes de nitroform ( $N$ lentement soluble);

(doses à l'hectare : $70 \mathrm{~kg}$ de $\mathbf{K}_{2} 0,200 \mathrm{~kg}$ de $\mathbf{P}_{2} 05,100 \mathrm{~kg}$ de $\mathrm{N}$ ).

La plantation a été effectuée dans les cuves au printemps 1979. 13 cuves ont été plantées en chênes âgés de 3 ans $(2+1)$ et les 13 autres en frênes âgés de 2 ans $(2+0)$. Afin d'éviter d'éventuels effets microclimatiques liés à la situation sur le terrain, la répartition des espèces a été randomisée.

(1) Ces recherches ont été financées en partie par la D.G.R.S.T. 
Le dispositif est complété par l'installation : de tuyaux d'arrosage sur la périphérie interne de chaque cuve, de tubes d'accès pour un humidimètre gamma neutronique (Aussenac \& Granier, 1979) et d'un toit mis en place à $20 \mathrm{~cm}$ au-dessus de chaque cuve. Cette couverture est constituée de panneaux en matière plastique opaque ondulée et perforée d'orifices permettant le passage des tiges de chacun des plants. Un joint souple assure l'étanchéité à ce niveau. Cette couverture est destinée à maintenir le sol des cuves à l'abri des précipitations, afin de permettre le contrôle de l'approvisionnement en eau. Ce toit a une surface supérieure à celle des cuves; il est légèrement incliné pour permettre l'évacuation des eaux de pluie.

En 1979 et 1980, tous les arbres ont été cultivés à la capacité au champ. L'expérimentation proprement dite a débuté au printemps 1981. Pendant les deux premières années, les arbres se sont vigoureusement développés, donnant des ensembles à couvert fermé. Afin de ne garder que des plants en bon état de développement, les individus dominés ont été éliminés; au total ont été conservés dans chaque cuve : 21 arbres pour les chênes et 16 arbres pour les frênes.

Le protocole expérimental adopté pour chaque espèce est le suivant :

- une cuve est laissée sans couverture, afin d’être alimentée en eau par les précipitations naturelles;

- deux cuves sont maintenues en permanence à la capacité au champ par arrosages hebdomadaires (cuves C);

- dix cuves sont mises en condition de dessèchement (cuves S); chacune n'est réhydratée (ramenée à la capacité au champ) que lorsque le potentiel hydrique de base des plants correspondants dépasse - 19 bars. Le potentiel hydrique est évalué à l'aide d'une bombe à pression (Aussenac \& Granier, 1978).

Afin d'étudier le comportement des arbres, différentes mesures et opérations ont été effectuées chaque semaine:

- mesure du potentiel de base $\left(P_{1:}\right)$ d'un des plants de chacune des 26 cuves (il s'agit du potentiel hydrique des rameaux mesuré en fin de nuit) ;

- mesure de l'humidité du sol, à l'aide d'un humidimètre gamma neutronique. Les mesures ont été faites de $-15 \mathrm{~cm}$ à $-45 \mathrm{~cm}$, ce qui n'a permis d'évaluer l'humidité volumique que des 55 premiers centimètres du sol de chaque cuve, la configuration de l'appareil ne permettant pas de faire des mesures plus rapprochées du fond. Par différence entre les profils hydriques de deux dates successives, il a été possible d'évaluer le prélèvement d'eau effectué par les plants dans cette tranche de sol. On a fait l'hypothèse que le prélèvement total dans les cuves $(0,-80 \mathrm{~cm})$ est proportionnel à celui effectué de 0 à $-55 \mathrm{~cm}$. Dans la suite de ce travail, lorsqu'il sera question d'E.T.R., il s'agira du prélèvement correspondant à la tranche de 0 à $-55 \mathrm{~cm}$;

- remise à la capacité au champ des 4 cuves maintenues humides en permanence et des cuves soumises au dessèchement et ayant atteint ou dépassé - 19 bars (Potentiel de base). A partir de la quantité d'eau apportée en arrosage et de la quantité d'eau recueillie par écoulement, il est possible de calculer la quantité d'eau consommée entre deux arrosages successifs :
E.T.R. $=\mathrm{A}-\mathrm{D}$
A : arrosage
D : écoulement 


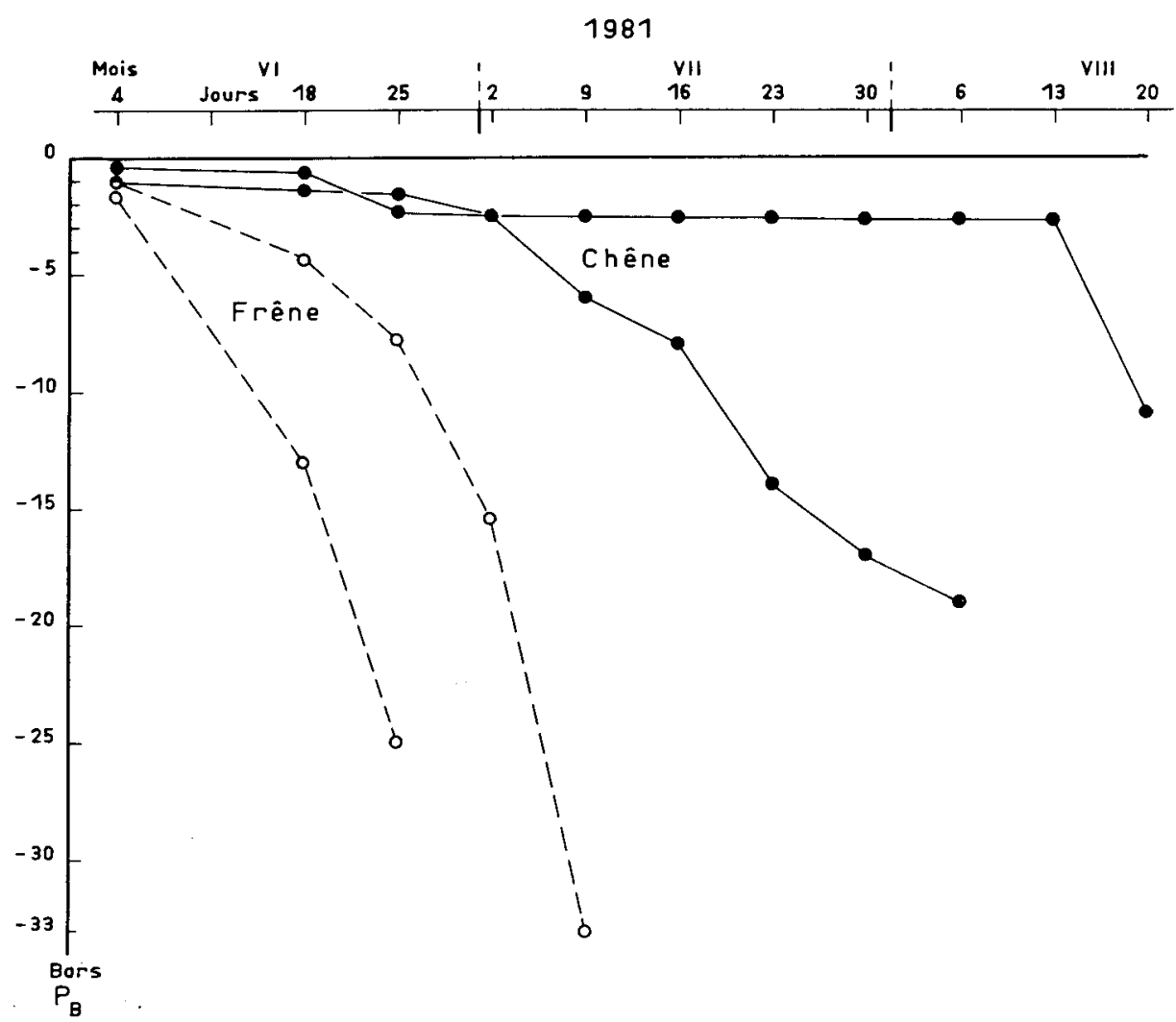

FIG. 1

Evolution du potentiel de base $\left(P_{B}\right)$, pour les cuves en phase de dessèchement : courbes extrêmes pour chacune des deux espèces.

Evolution of predawn potential $\left(P_{B}\right)$ for the trees during the dry period: extreme curves for each of those two species.

Il y a lieu de remarquer que, en raison du recouvrement des cuves par les plaques en matière plastique, la valeur mesurée de E.T.R. correspond presque exclusivement à la transpiration des plants;

- mesure de la croissance en hauteur de la pousse terminale de tous les plants.

Afin de préciser le comportement hydrique des plants, il a été effectué en juillet 1981 une étude de la cinétique journalière du potentiel hydrique et de la résistance stomatique des plants de certaines cuves sélectionnées de manière à présenter une gamme très étalée des potentiels de base. Les mesures de résistance stomatique $\left(r_{\mathrm{S}}\right)$ ont été effectuées à l'aide d'un poromètre à diffusion Van Bavel, modèle KANEMASU et al. (1969).

Pour les 20 cuves soumises au dessèchement, l'arrêt des arrosages a été appliqué pour chaque espèce lorsque le débourrement a été acquis pour l'ensemble des plants. Le frêne avait commencé à débourrer plus précocement que le chêne, mais par 
suite de dégâts occasionnés par une gelée tardive, il en est résulté un débourrement concomitant des deux essences. La croissance en hauteur des plants a donc démarré en même temps.

\section{Résultats}

\subsection{Evolution comparée de l'état hydrique du chêne et du frêne en phase de dessèchement}

La figure 1 montre l'évolution des potentiels de base $\left(\mathrm{P}_{\mathrm{B}}\right)$ (courbes extrêmes : dessèchement le plus rapide et dessèchement le plus lent) pour chacune des deux espèces en fonction du temps. Cette figure ne commence qu'au 4-06-1981, car ce n'est qu'à partir de cette date que la diminution des potentiels de base devient sensible, du moins pour le frêne. On constate que la diminution du potentiel hydrique de base est beaucoup plus rapide chez le frêne que chez le chêne. Le tableau 1 indique le temps mis par les deux espèces dans chaque cuve pour atteindre différents niveaux de stress hydrique. En moyenne, pour atteindre - 10 bars, les frênes ont mis 29 jours, contre 69 jours poụr les chênes. Il y a lieu d'ajouter que, compte tenu de cette vitesse d'évolution du potentiel de base, les frênes ont été réhydratés 3 fois, alors que les chênes ne l'ont été qu'une fois, en fin d'été. Bien qu'il ne soit pas possible de faire une moyenne des consommations en eau, puisque les réalimentations n'ont pas été effectuées exactement au même niveau de stress hydrique, il est intéressant de noter que toutes les cuves de frênes ont consommé beaucoup plus d'eau que les cuves de chêne (tabl. 2). Pour les cuves soumises à la capacité au champ, on constate des différences, les frênes ayant eu dans ce cas aussi une consommation supérieure aux chênes, mais ces différences sont proportionnellement beaucoup moins importantes que dans le cas des cuves en dessèchement.

\section{Tableau 1}

Temps (jour) mis pour atteindre un niveau de stress déterminé chez le chêne et le frêne (à partir du début du dessèchement le 22-4-1981).

Time (day) elapsed to reach a definite level of predawn potential from the beginning of the drying period (22-4-1981).

\begin{tabular}{|c|c|c|c|c|c|c|c|}
\hline \multicolumn{4}{|c|}{ Chêne } & \multicolumn{4}{|c|}{ Frêne } \\
\hline \multirow{2}{*}{$\mathrm{N}^{\circ}$ cuve } & \multicolumn{3}{|c|}{$P_{B}$ (bar) } & \multirow{2}{*}{$\mathrm{N}^{\circ}$ cuve } & \multicolumn{3}{|c|}{$P_{B}$ (bar) } \\
\hline & -10 & -20 & -30 & & -10 & -20 & -30 \\
\hline 4 & 68 & 84 & & 2 & 18 & 2.5 & \\
\hline 5 & 58 & 71 & & 3 & 30 & 37 & \\
\hline 8 & 64 & 80 & & 11 & 37 & 39 & 42 \\
\hline 9 & 53 & 75 & & 12 & 31 & 37 & \\
\hline 10 & 75 & & & 13 & 31 & 37 & 43 \\
\hline 17 & 81 & & & 14 & 18 & 26 & \\
\hline 18 & 81 & & & 15 & 23 & 31 & \\
\hline 19 & 88 & & & 16 & 31 & 37 & 42 \\
\hline 21 & 53 & 84 & & 20 & 31 & 37 & 42 \\
\hline 23 & 71 & 81 & & 25 & 37 & 40 & 44 \\
\hline Moyenne & 69,2 & & & Moyenne & 28,7 & 34,6 & \\
\hline
\end{tabular}




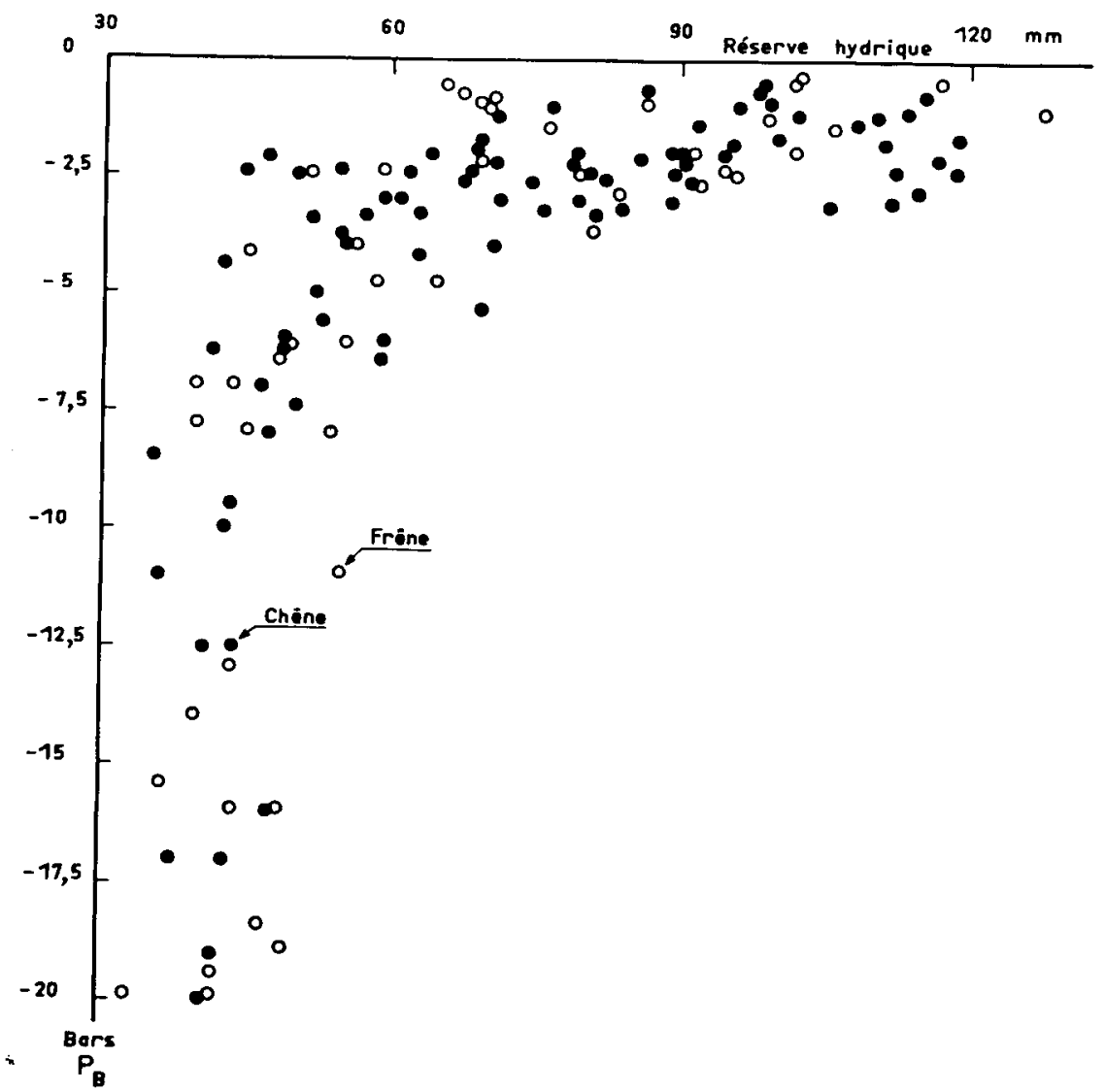

FIG. 2

Relation entre potentiel de base $\left(P_{B}\right)$ et réserve hydrique du sol $(0 \grave{a}-55 \mathrm{~cm})$. Relation between predaw'n potential $\left(P_{B}\right)$ and water reserve of soil $(0-55 \mathrm{~cm})$.

En raison de la texture sableuse du sol, le potentiel de base des plants ne commence à baisser véritablement que lorsque la réserve hydrique du sol (mesurée sur $55 \mathrm{~cm}$ d'épaisseur) devient inférieurc à $60 \mathrm{~mm}$, ce qui représente environ 45 p. 100 de la réserve à la capacité au champ (fig. 2). Le phénomène est identique pour les deux espèces étudiées; ceci confirme d'ailleurs l'intérêt de l'utilisation du $P_{\mathrm{B}}$ pour définir l'état hydrique du sol dans la sphère radiculaire.

\subsection{Influence de la disponibilité en eau sur l'évapotranspiration des deux espèces}

Les résultats précédents montrent que le frêne consomme beaucoup plus rapidement l'eau disponible dans le sol que le chêne. Afin d'essayer de comprendre le fonctionnement de ces deux essences en phase de dessèchement, on a étudié la relation entre l'évapotranspiration réelle et la réserve hydrique, pour les différentes cuves. Il s'agit 


\section{TableaU 2}

\section{Consommation en eau des cuves de chêne et de frêne} pendant la période du 22-4-1981 au 28-9-1981

Water consumption in oak and ash containers from 22-4-1981 to 28-9-1981.

\begin{tabular}{|c|c|c|c|c|c|}
\hline \multicolumn{3}{|c|}{ Chêne } & \multicolumn{3}{|c|}{ Frêne } \\
\hline $\mathrm{N}^{\circ}$ de cuve & Traitement & $\begin{array}{l}\text { Consommation } \\
\text { en eau } \\
\text { (litre) }\end{array}$ & $\mathrm{N}^{\circ}$ de cuve & Traitement & $\begin{array}{c}\text { Consommation } \\
\text { en eau } \\
\text { (litre) }\end{array}$ \\
\hline 7 & $\begin{array}{l}\text { Capacité } \\
\text { au champ }\end{array}$ & 1350 & 1 & $\begin{array}{l}\text { Capacité } \\
\text { au champ }\end{array}$ & 1517 \\
\hline 22 & $\begin{array}{l}\text { Capacité } \\
\text { au champ }\end{array}$ & 1200 & 24 & $\begin{array}{l}\text { Capacité } \\
\text { au champ }\end{array}$ & 1410 \\
\hline 4 & Dessèchement & 430 & 2 & Dessèchement & 791 \\
\hline 5 & Dessèchement & 495 & 3 & Dessèchement & 813 \\
\hline 8 & Dessèchement & 489 & 11 & Dessèchement & 736 \\
\hline 9 & Dessèchement & 512 & 12 & Dessèchement & 745 \\
\hline 10 & Dessèchement & 482 & 13 & Dessèchement & 802 \\
\hline 17 & Dessèchement & 437 & 14 & Dessèchement & 730 \\
\hline 18 & Dessèchement & 527 & 15 & Dessèchement & 791 \\
\hline 19. & Dessèchement & 461 & 16 & Dessèchement & 824 \\
\hline 21 & Dessèchement & 516 & 20 & Dessèchement & 942 \\
\hline 23 & Dessèchement & 537 & $25^{*}$ & Dessèchement & 624 \\
\hline
\end{tabular}

(*) Cuve particulièrement atteinte par les gelées tardives et très retardée dans son développement.

en fait du prélèvement d'eau et de la réserve hydrique dans les 55 premiers centimètres du sol. A titre d'exemple, la figure 3 représente cette relation pour la semaine du 18 au 25 juin 1981. On constate que, dès que le sol se dessèche légèrement, le chêne diminue fortement sa transpiration; par contre le frêne ne la diminue sensiblement que pour des réserves hydriques beaucoup plus faibles. Si l'on considère d'autres périodes, les résultats sont comparables. Ce phénomène explique que le frêne consomme beaucoup plus rapidement les réserves en eau que le chêne. Cette diminution d'E.T.R. du chêne ne s'accompagne pas d'une réduction immédiate de la croissance en hauteur. D'une façon générale, pour l'ensemble de la période d'étude, on constate que le frêne ne commence à diminuer son évapotranspiration que lorsque la réserve en eau du sol est très abaissée 
(environ 60 p. 100 de la réserve à la capacité au champ), alors que pour le chêne une forte baisse est déjà acquise pour une réserve encore élevée (inférieure de 13 p. 100 à la capacité au champ); le niveau d'humidité du sol correspondant au début de la diminution d'E.T.R. n’a pu être déterminé car nous ne disposons pas de données correspondantes.

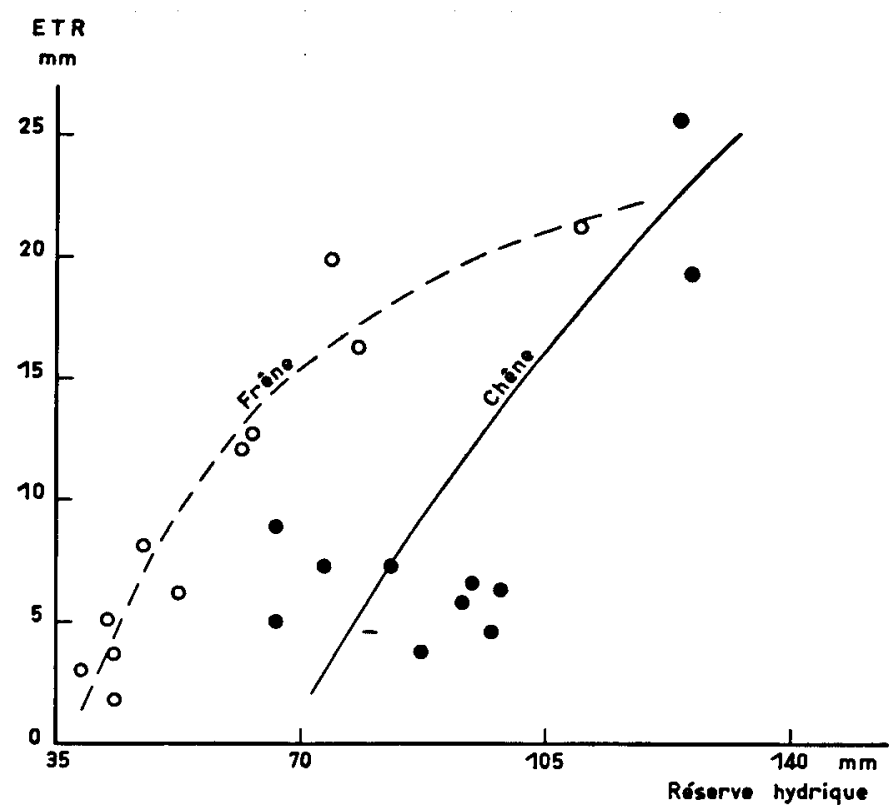

Fig. 3

Evapotranspiration (E.T.R.) en fonction de la réserve hydrique

dans les différentes cuves $(0$ a $-55 \mathrm{~cm})$ au cours de la semaine du 18 au 25-6-1981.

Evapotranspiration (E.T.R.) as a function of water reserve of different containers during the week from 18 to 25 june 1981 .

Ces résultats expliquent sans doute que, même dans le traitement qualifié de capacité au champ, le chêne consomme moins d'eau que le frêne, compte tenu d'un rythme hebdomadaire probablement trop faible de réalimentation en eau.

L'étude des relations existant au cours d'une journée (9 juillet 1981) entre potentiel hydrique $\left(\Psi_{f}\right)$ et résistance stomatique $\left(r_{s}\right)$ explique les résultats précédents et fait apparaître un comportement très différent des deux espèces étudiées (fig. 4). En effet, pour une même chute du potentiel hydrique, la résistance stomatique augmente plus vite chez le chêne que chez le frêne. On constate aussi que lorsque le potentiel atteint - 14 bars chez le chêne, la résistance stomatique augmente considérablement, alors que cela ne se produit chez le frêne que pour des valeurs comprises entre -30 et -35 bars. 
Ce phénomène explique donc la raison pour laquelle le chêne commence à réduire sa transpiration pour des réserves en eau plus élevées que ne le fait le frêne. On comprend alors pourquoi le sol se dessèche moins vite dans les cuves occupées par le chêne. En conditions naturelles, ce dernier résistera donc plus longtemps au cours d'une période de sécheresse.

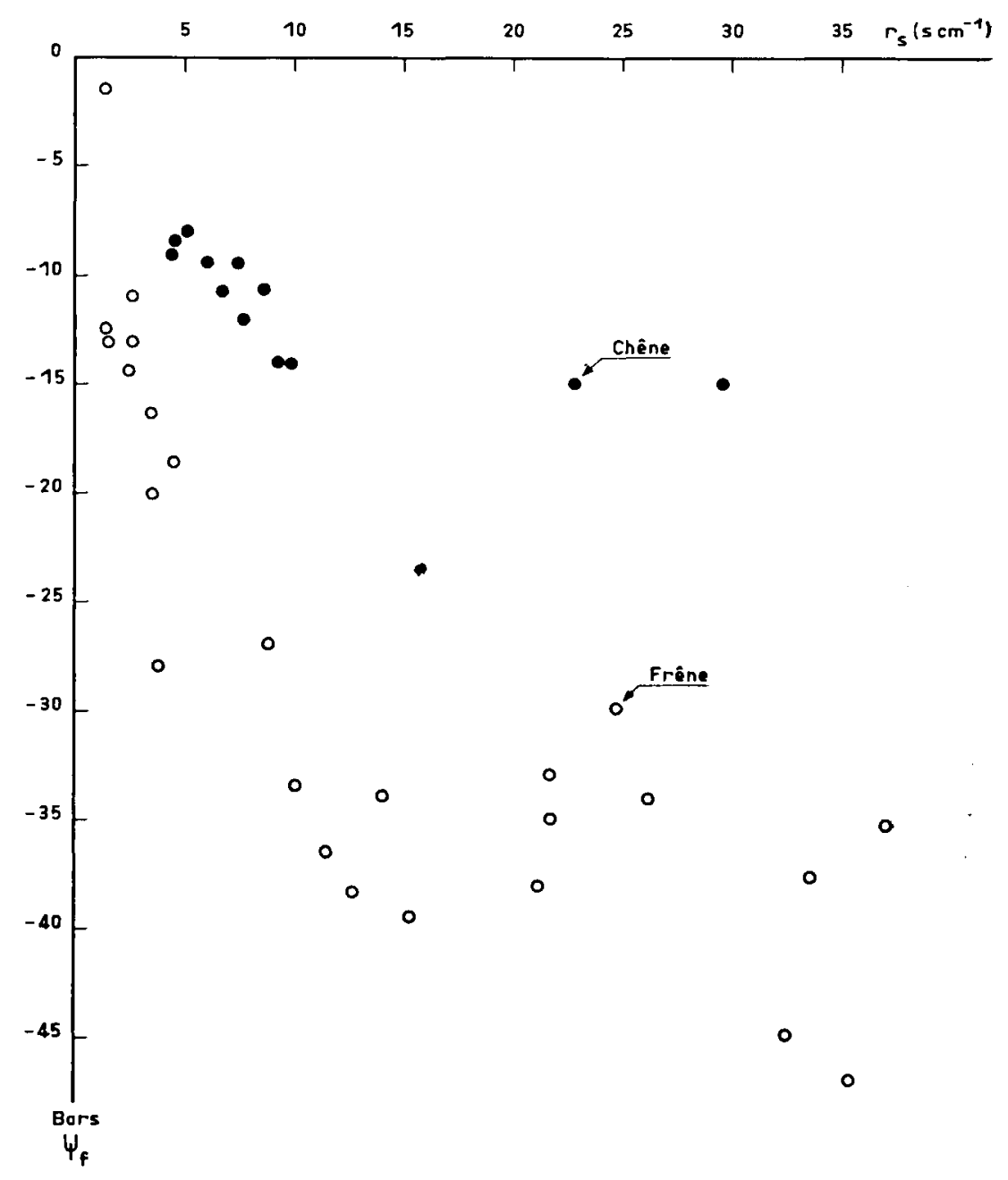

FIG. 4

Relation entre le potentiel hydrique des rameaux (w) et la résistance stomatique $\left(r_{s}\right)$, (mesures effectuées sur un certain nombre de plants

à différentes reprises tout au long d'une journée ensoleillée : 9-7-1981).

Relation between xylem water potential of twigs ( $y, f)$ and the stomatal resistance $\left(r_{s}\right)$ throughout a sunny day (7-9-1981). 


\subsection{Influence $d u$ dessèchement du sol sur la croissance en hauteur des deux espèces}

La figure 5 rapporte l'évolution de l'accroissement cumulé en hauteur. On observe d'une part que le frêne n'a eu qu'une seule poussée de croissance, alors que le chêne en a eu trois dans le traitement à la capacité au champ et deux pour les cuves subissant un dessèchement, la première mesure (27-5-1981) ayant été effectuée alors que la première pousse du chêne était terminée. Dans les cuves à la capacité au champ, les frênes ont eu une croissance en hauteur plus forte que les chênes, mais ces derniers ont des ramifications latérales beaucoup plus nombreuses et denses qui font que, au total, la biomasse foliaire des deux espèces apparaît très comparable globalement au niveau d'une cuve. On remarque aussi que les cuves en phase de dessèchement ont moins poussé que les cuves bien arrosées. L'examen de l'allongement relatif des pousses en fonction du potentiel de base (fig. 6) montre que chez les deux espèces la croissance en hauteur est arrêtée au-delà de -11 bars.

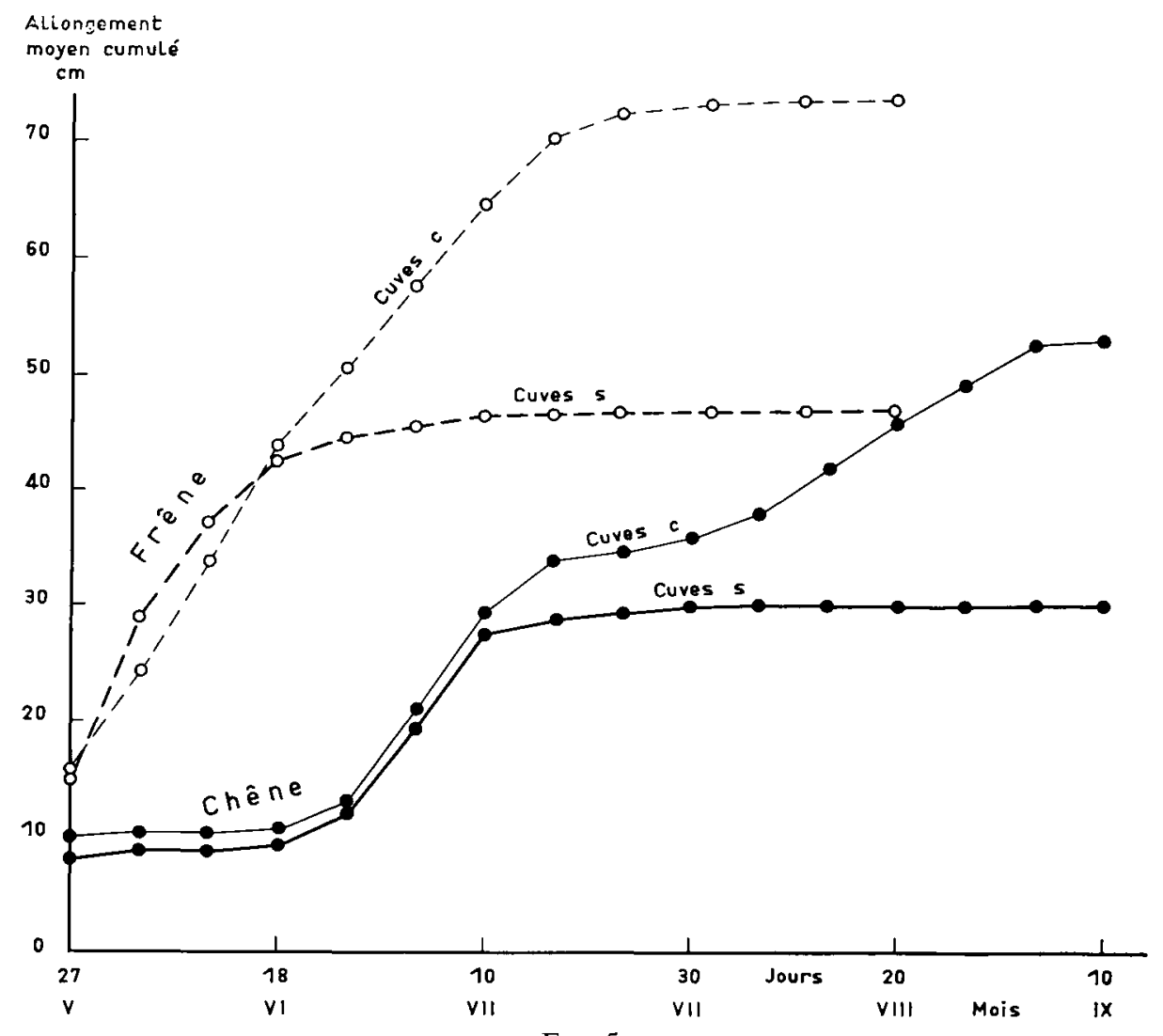

FIG. 5

Evolution de la longueur moyenne de la pousse terminale des plants pour les cuves à la capacité au champ (C) et celles soumises au dessèchement (S) en 1981. Evolution of the average lenght of plants terminal shoot for trees on soils at field capacity $(C)$ and those on drying soils (S) in 1981. 
En fin de saison (fig. 5), la diminution relative de croissance due à la sécheresse est très comparable pour les deux espèces, mais il ne faut pas oublier que les cuves «sèches» de frêne ont reçu beaucoup plus d'eau que les cuves correspondantes de chêne; rappelons en effet que la réhydratation des cuves s'effectuait quand le potentiel de base atteignait ou dépassait - 19 bars. A ce niveau de sécheresse, la réalimentation était nécessaire, car les feuilles de frêne montraient des signes nets de flétrissement.

\section{Discussion et conclusion}

L'étude entreprise ici a permis de caractériser comparativement le chêne pédonculé et le frêne. Des différences importantes de comportement ont été mises en évidence. Le frêne peut être considéré comme une espèce grosse consommatrice d'cau dans la mesure où elle ne contrôle que très tardivement sa transpiration. Ce comportement a pour conséquence, toutes choses égales par ailleurs, d'amener cette espèce à des stress hydriques élevés beaucoup plus rapidement que le chêne. Dans l'hypothèse d'une alimentation en eau déficiente, le frêne se trouve donc beaucoup plus précocement en position difficile que le chênc.
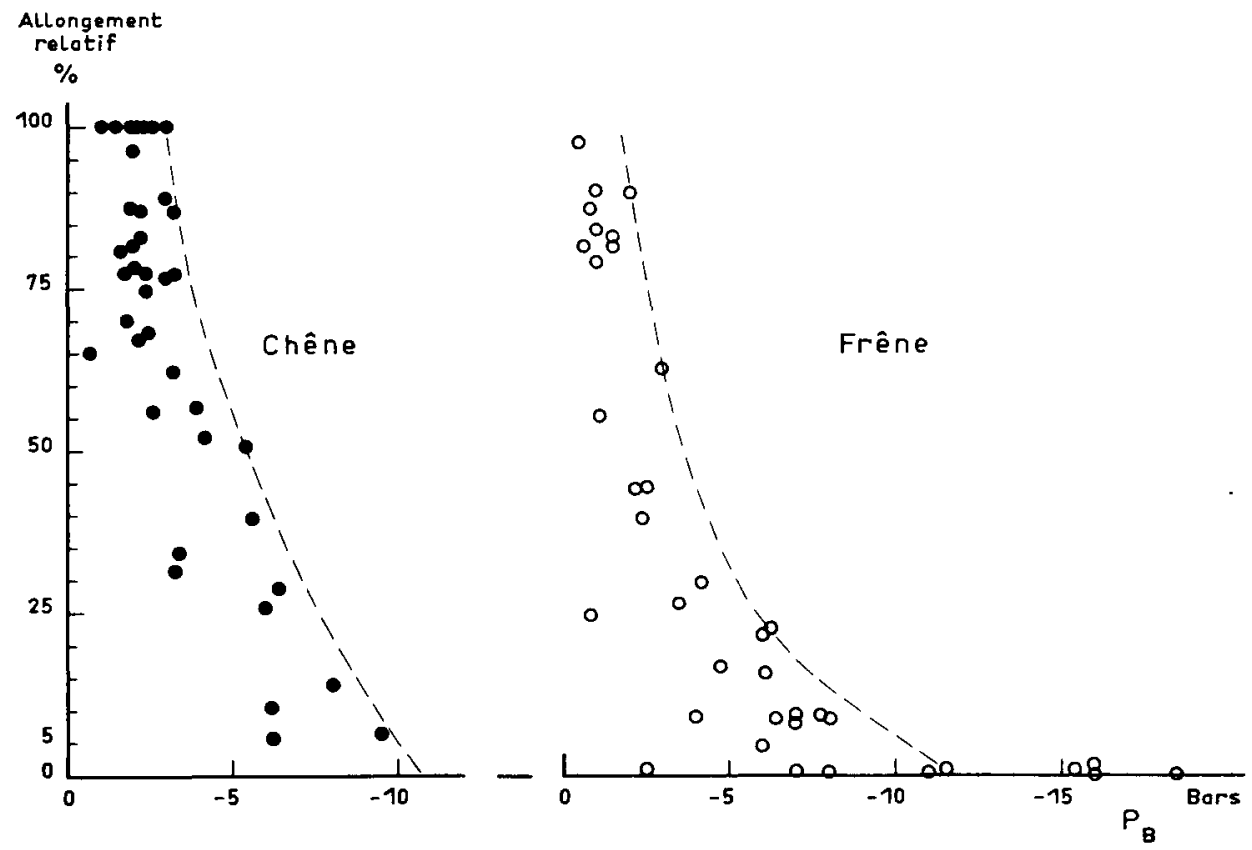

FIG. 6

Allongement hebdomadaire moyen des plants pour chacune des cuves soumises au dessèchement (exprimé en pourcentage de l'allongement moyen des plants pour les cuves à la capacité au champ) en fonction du potentiel de base $\left(P_{B}\right)$.

Average height growth per week of plants for each container during the drying period (as a percentage of the average growth of plants on soil at field capacity) as a function of predawn potential $\left(\boldsymbol{P}_{B}\right)$. 
Cette capacité transpiratoire élevée du frêne a été également notée par BrauN (1977), qui trouve que cette espèce a une transpiration plus élevée que laulne glutineux et les grands érables. KozLowsk et al. (1974), étudiant Acer saccharum et Fraxinus americana, observent aussi une transpiration plus élevée du frêne américain ; ils attribuent cette capacité transpiratoire importante à des stomates plus grands, mais beaucoup moins nombreux. Les comptages effectués sur les deux espèces étudiées ici indiquent pour Fraxinus excelsior 175 stomates $/ \mathrm{mm}^{2}$, et pour Quercus pedunculata 500 stomates $/ \mathrm{mm}^{2}$, mais il ne semble pas y avoir de grandes différences en ce qui concerne la taille des stomates.

Le chêne apparaît comme une espèce micux adaptée à des situations moins favorisées sur le plan hydrique, dans la mesure où elle contrôle précocement les échanges transpiratoires. Ce comportement permet à cette essence d'avoir une amplitude écologique plus grande que te frêne.

Les résultats obtenus ici (possibilité de 2 ou 3 pousses successives selon le niveau d'alimentation en eau) indiquent cependant que le facteur hydrique est un élément important de la productivité de cette essence.

Pour les deux espèces, l'arrêt de la croissance en hauteur se produit à un niveau de stress hydrique relativement faible : - 11 bars, si on le compare à d'autres espèces ; Aussenac \& Finkelstein (1982) trouvent en effet - 21 bars pour le cèdre de l'Atlas.

Bien qu'en valeur absolue, la croissance en hauteur initiale du frêne soit supérieure à celle du chêne, ce dernier a la possibilité de pousser, si les conditions d'alimentation le permettent, de mi-mai à mi-septembre, alors que le frêne a une période de croissance en hauteur plus limitée, de mi-mai à fin juillet.

Au plan sylvicole, les résultats obtenus ici permettent de préciser les limites du frêne. Il s'agit d'une espèce dont l'utilisation ne peut être envisagée que dans des situations à disponibilités en cau importantes (présence d'une nappe phréatique ou sol profond ou bas de pente). Dans les zones à bilan hydrique déficitaire, l'avenir des arbres est compromis. Ces résultats recoupent les travaux de Devauchelle \& Levy (1977), qui indiquent qu'en Lorraine lalimentation en eau est le principal facteur limitant la production du frêne. Notons aussi qu'en peuplement, il est classique d'observer sur plateaux calcaires des régénérations importantes de frêne qui disparaissent rapidement. Dans la mise en valeur des taillis et taillis-sous-futaie, il est certain que le frêne ne peut être cnvisagé que dans des situations bien particulières.

Reçu pour publication le 14 novembre 1982.

\section{Summary}

Influence of soil drying on the plant water relations and growth of pedunculate oak (Quercus pedunculata Ehrl.) and ash (Fraxinus excelsior L.)

We compared experimentally the plant-water relations of pedunculate oak and ash submitted to drying cycles (no water supply was brought until the predawn potential $\left(\mathrm{P}_{\mathrm{B}}\right)$ reached under - 19 bars in buried containers). The plants were respectively 5 and 4 years old. 
The oak transpiration decreased a lot as soon as the soil dried slightly; on the contrary, the ash transpiration clearly decreased only for soil water contents much less important. Indeed, for a similar decreasing of the xylem water potential during the day, the stomatal resistance increased faster for the oak than for the ash ; moreover, a considerable increasing of stomatal resistance occured when this xylem water potential reached -14 bars in the oak, but only - 30 bars in the Ash.

Thus, the decreasing of the $P_{1:}$ and the soil drying were much faster for the Ash then for the oak.

The height growth was stopped, for both species, as soon as the $P_{n}$ was lower than - 11 bars; this value was reached much sooner for the ash on account of his important transpiration.

Thus, the oak is seen as a species better adapted than the Ash to forest sites poorly supplied in water; so its ecological amplitude is much more important.

\section{Références bibliographiques}

Aussenac G., Granier A., 1978. Quelques résultats de cinétique journalière du potentiel de sève chez les arbres forestiers. Ann. Sci. for. 35 (1), 19-32.

Aussenac G., Granier A., 1979. Etude bioclimatique d'une futaie feuillue (Fagus silvatica $\mathrm{L}$. et Quercus sessiliflora Salisb.) de l'Est de la France. II. Etude de l'humidité du sol et de l'évapotranspiration réelle. Ann. Sci. for. 36 (4), 265-280.

Aussenac G., Finkelstein D., 1983. Influence de la sécheresse sur la croissance et la photosynthèse du cèdre. Ann. Sci. for., 40 (1), 67-77.

Braun H.J., 1977. Growth and water economy of the trees Acer platanoiddes L., Acer pseudoplatanus L., and Fraxinus excelsior L. Z. Pflanzenphysiol., 84 (5), 459-462.

Davies W.J., Kozlowski T.T., 1975. Stomatal response to changes in light intensity as influenced by plant water stress. For. Sci., 21 (2), 129-133.

Devauchelle R., Levy G., 1977. Proprićtés stationnelles et croissance du frêne dans l'Est de la France, étude de certaines caractéristiques de cette essence. Ann. Sci. for., 34 (3), 231-244.

Kanemasu E.T., Thurtell G.W., Tanner C.B., 1969. Design, calibration and lield use of a stomatal diffusion parometer. Plant Physiol., 44, 881-885.

Kozlowski T.T., Davies W.J., Carlson S.D., 1974. Transpiration rates of Fraxinus americana and Acer saccharum leaves. Can. J. for. Res., 3 (4), 259-267.

Le Goff N., Levy G., 1983. Etude des relations station-productivité pour le frêne dans la région Nord-Picardie. Ann. Sci. for. (à paraître). 